open ${ }^{\text {De Grer }}$ ACta Univ. SApientiae, Informatica 9, 2 (2017) 119-133

DOI: $10.1515 /$ ausi-2017-0008

\title{
Further results on color energy of graphs
}

\author{
Prajakta Bharat JOSHI \\ Christ University \\ Bengaluru - 560029, India. \\ email: \\ prajakta.joshi@res.christuniversity.in
}

\author{
Mayamma JOSEPH \\ Department of Mathematics \\ Christ University \\ Bengaluru - 560029, India.
}

email:

\begin{abstract}
Given a colored graph $G$, its color energy $E_{c}(G)$ is defined as the sum of the absolute values of the eigenvalues of the color matrix of G. The concept of color energy was introduced by Adiga et al. [1]. In this article, we obtain some new bounds for the color energy of graphs and establish relationship between color energy $E_{\mathfrak{c}}(G)$ and energy $E(G)$ of a graph G. Further, we construct some new families of graphs in which one is non-co-spectral color-equienergetic with some families of graphs and another is color-hyperenergetic. Also we derive explicit formulas for their color energies.
\end{abstract}

\section{Introduction}

The concept of energy of a graph $\mathrm{G}$ was introduced by Gutman [9] in 1978 as the sum of the absolute values of the eigenvalues of the adjacency matrix of the graph $\mathrm{G}$. His focus was to solve a question from theoretical chemistry "how energy depends on the molecular structure?" In other words, he tried to find the relation between the energy of a graph and its structure. Gutman has

Computing Classification System 1998: G.2.2

Mathematics Subject Classification 2010: 05C15, 05C50

Key words and phrases: energy, color matrix, color eigenvalues, color energy of a graph 
obtained an upper and a lower bound for energy of a graph $\mathrm{G}$ in terms its size $\mathrm{m}$,

$$
2 \sqrt{\mathrm{m}} \leq \mathrm{E}(\mathrm{G}) \leq 2 \mathrm{~m}
$$

He further characterized graphs for which these bounds are sharp. For details of graph energy we refer to $[9,10,11]$.

For many years, researchers have extended the concept of graph energy and continued to work on varieties of graph energy such as Laplacian energy, distance energy etc [11].

Recently Adiga et al. [1] introduced the concept of color energy of a graph based on the color matrix of the graph.

Definition 1 Let $\mathrm{G}$ be a vertex colored graph of order $\mathrm{n}$. Then the color matrix of $\mathrm{G}$ is the matrix $\mathrm{A}_{\mathrm{c}}(\mathrm{G})=\left[\mathrm{a}_{\mathrm{ij}}\right]_{\mathfrak{n} \times \mathrm{n}}$, whose entries are given by

$$
\mathrm{a}_{i j}= \begin{cases}1 & \text { if } v_{i} \text { and } v_{j} \text { are adjacent with } \mathrm{c}\left(v_{i}\right) \neq \mathrm{c}\left(v_{j}\right) \\ -1 & \text { if } v_{i} \text { and } v_{j} \text { are non-adjacent with } \mathrm{c}\left(v_{i}\right)=\mathrm{c}\left(v_{j}\right) \\ 0 & \text { otherwise. }\end{cases}
$$

where $\mathrm{c}\left(v_{i}\right)$ is the color of a vertex $v_{i}$ in $\mathrm{G}$.

If the eigenvalues of $A_{c}(G)$ are $\lambda_{1}, \lambda_{2}, \ldots \lambda_{n}$, which are also called as color eigenvalues, then the color energy $E_{c}(G)$ is the sum of their absolute values. That is,

$$
\mathrm{E}_{\mathrm{c}}(\mathrm{G})=\sum_{i=1}^{\mathrm{n}}\left|\lambda_{i}\right|
$$

If a graph $G$ is colored with minimum number of colors $\chi$, then $E_{\chi}(G)$ is the color energy of $G, A_{\chi}(G)$ is the color matrix, $P_{\chi}(G, \lambda)$ is the characteristic polynomial and $\operatorname{Spec}_{\chi}(G)$ is the spectrum of the graph $G$.

They have proved that

$$
\sum_{i=1}^{n} \lambda_{i}^{2}=2\left(m+m_{c}^{\prime}\right)
$$

where $m$ is the size of $G$ and $m_{c}^{\prime}$ is the number of pairs of non-adjacent vertices receiving the same color in $\mathrm{G}$.

Further, the authors have derived explicit formulas for color energies of some families of graphs and have obtained bounds for $E_{c}(G)$. Among those results the following would be used for further discussion. 
The color energies of $K_{n}$ and $K_{1, n-1}$ are

$$
E_{\chi}\left(K_{n}\right)=2(n-1) \text { and } E_{\chi}\left(K_{1, n-1}\right)=2(n-1)
$$

respectively with the spectra

$$
\operatorname{Spec}_{\chi}\left(K_{n}\right)=\left(\begin{array}{cc}
-1 & n-1 \\
n-1 & 1
\end{array}\right) \text { and } \operatorname{Spec}_{\chi}\left(K_{1, n-1}\right)=\left(\begin{array}{cc}
-(n-1) & 1 \\
1 & n-1
\end{array}\right)
$$

and for any graph G

$$
\sqrt{2\left(m+m_{c}^{\prime}\right)+n(n-1) D^{\frac{2}{n}}} \leq E_{c}(G) \leq \sqrt{2 n\left(m+m_{c}^{\prime}\right)}
$$

where $D=\left|\operatorname{det}\left(A_{c}(G)\right)\right|$.

In this direction, Rajesh Kanna et al. [12] have proved that the color energy of the friendship graph $F_{3}^{(k)}$ of order $n$ is $2(n-1)$ with the spectrum

$$
\operatorname{Spec}_{\chi}\left(F_{3}^{(k)}\right)=\left(\begin{array}{ccc}
0 & 2 & -k \\
k-1 & k & 2
\end{array}\right)
$$

where the friendship graph $F_{3}^{(k)}$ is the graph obtained by taking $k$ copies of $C_{3}$ with a vertex in common.

Research in the area of color energy has seen rapid rise in recent years and concepts such as color Laplacian energy $[4,15]$, color signless Laplacian energy [5], minimum covering color energy of a graph [13], reduced color energy etc. $[2,3]$ were added to the literature. Although several studies have been done in this area, no study has been initiated to explore the relation between color energy and energy of graphs.

Apart from these studies, a classification of graphs of order $\leq 6$ on the basis of their color energy is found in [16]. Also the lower bounds in terms of the smallest and largest color eigenvalues of a graph $G$ with order $n$, size $m$ and the number of pairs of the non-adjacent vertices in $G$ receiving same color were obtained in [17].

In this paper, we establish relationships between $E_{c}(G)$ and $E(G)$ apart from finding new bounds for $E_{c}(G)$. In addition to this, we introduce non-cospectral graphs that are color-equienergetic with complete graphs and a family of color-hyperenergetic graphs.

All graphs considered in this paper are simple and connected. Our graph theoretic and spectral graph theoretic terminologies follow $[6,7,8,18]$. 


\section{Bounds for color energy of graphs}

In this section, we present some new bounds for the color energy of graphs in terms of the largest positive color eigenvalue $\lambda_{1}$ and the largest absolute value of the color eigenvalue $\lambda_{\max }$ of $A_{\mathcal{c}}(G)$. First we present an upper bound for color energy in terms of $\lambda_{1}$, order $n$, size $m$ and $m_{c}^{\prime}$ which denotes the number of pairs of non-adjacent vertices receiving the same color in $\mathrm{G}$.

Theorem 2 Let $\mathrm{G}$ be a colored graph of order $\mathrm{n}$, size $\mathrm{m}$. Let $\lambda_{1} \geq \lambda_{2} \geq \cdots \geq$ $\lambda_{\mathrm{n}}$ be the color eigenvalues of $\mathrm{A}_{\mathrm{c}}(\mathrm{G})$. Then

$$
E_{c}(G) \leq\left|\lambda_{1}\right|+\sqrt{(n-1)\left[2\left(m+m_{c}^{\prime}\right)-\lambda_{1}^{2}\right]}
$$

where $\mathrm{m}_{\mathrm{c}}^{\prime}$ be the number of pairs of non-adjacent vertices receiving the same color.

Proof. $\lambda_{1} \geq \lambda_{2} \geq \cdots \geq \lambda_{n}$ are color eigenvalues of $A_{c}(G)$, so by the CauchySchwartz inequality,

$$
\begin{aligned}
\left(\sum_{i=2}^{n}\left|\lambda_{i}\right|\right)^{2} & \leq(n-1)\left(\sum_{i=2}^{n}\left|\lambda_{i}\right|^{2}\right) \\
\sum_{i=2}^{n}\left|\lambda_{i}\right| & \leq \sqrt{(n-1)\left(\sum_{i=2}^{n}\left|\lambda_{i}\right|^{2}\right)} \\
& =\sqrt{(n-1)\left[2\left(m+m_{c}^{\prime}\right)-\lambda_{1}^{2}\right]}, \text { by Equation }(2) .
\end{aligned}
$$

Hence,

$$
E_{c}(G) \leq\left|\lambda_{1}\right|+\sqrt{(n-1)\left[2\left(m+m_{c}^{\prime}\right)-\lambda_{1}^{2}\right]} .
$$

Remark 3 The above theorem gives an upper bound for color energy of a graph G. This is an improvement on the upper bound given in the Inequality (5). For example, consider the graph $\mathrm{G}$ given in Figure 1 which is a paw. $\chi(\mathrm{G})=3$ with respect to given coloring. Inequality (5) yields the result that $\mathrm{E}_{\mathrm{c}}(\mathrm{G}) \leq 6.33$ whereas Inequality (7) shows that $\mathrm{E}_{\mathrm{c}}(\mathrm{G}) \leq 6.24$. It is to be observed that $\operatorname{Spec}_{\chi}(\mathrm{G})=\left(\begin{array}{cccc}-2 & -1 & 1 & 2 \\ 1 & 1 & 1 & 1\end{array}\right)$ and hence $\mathrm{E}_{\chi}(\mathrm{G})=6$. 


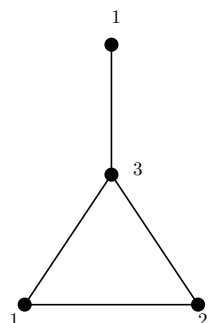

Figure 1: Graph G

Theorem 4 If $\mathrm{G}$ is a graph of order $n$, size $m$ and $\lambda_{\max }$ is the largest absolute value of eigenvalue of the color matrix of $\mathrm{G}$, then

$$
E_{c}(G) \geq\left(\frac{2\left(m+m_{c}^{\prime}\right)}{\lambda_{\max }}\right)
$$

where $\mathrm{m}_{\mathrm{c}}^{\prime}$ be the number of pairs of non-adjacent vertices receiving the same color.

Proof. Let $\lambda_{\max }$ be the largest absolute value of color eigenvalue of $A_{c}(G)$. Then

$$
\lambda_{\max }\left|\lambda_{i}\right| \geq \lambda_{i}^{2}
$$

holds for $i=1,2, \ldots, n$. Then summing over all i's, we get

$$
\begin{aligned}
& \sum_{i=1}^{n} \lambda_{\max }\left|\lambda_{i}\right| \geq \sum_{i=1}^{n} \lambda_{i}^{2} \\
& \lambda_{\max } \sum_{i=1}^{n}\left|\lambda_{i}\right| \geq 2\left(m+m_{c}^{\prime}\right), \text { by Equation (2). }
\end{aligned}
$$

So that,

$$
E_{c}(G) \geq\left(\frac{2\left(m+m_{c}^{\prime}\right)}{\lambda_{\max }}\right)
$$

Next we present bounds of the color energy of a graph $G$ in terms of only $\mathrm{m}$ and $\mathrm{m}_{\mathrm{c}}^{\prime}$. In order to prove this result, we require the following lemma.

Lemma 5 ([14]) If $A$ is a real or complex $\mathrm{n} \times \mathrm{n}$ matrix with eigenvalues $\lambda_{1}, \lambda_{2}, \ldots, \lambda_{n}$, then for $1 \leq \mathrm{k} \leq \mathrm{n}$ 
1. $S_{k}=(-1)^{k} c_{k}$.

2. $\mathrm{S}_{\mathrm{k}}$ is the sum of the $\mathrm{k} \times \mathrm{k}$ principal minors of $A$.

where $c_{\mathrm{k}}$ s are the coefficients in characteristic polynomial of $A$ and $S_{\mathrm{k}}$, the $\mathrm{k}^{\text {th }}$ symmetric function of $\lambda_{1}, \lambda_{2}, \ldots, \lambda_{n}$ is the sum of the products of the eigenvalues taken $\mathrm{k}$ at a time.

Theorem 6 If $\mathrm{G}$ is a colored graph of order $\mathrm{n}$, size $\mathrm{m}$ and $\mathrm{m}_{\mathrm{c}}^{\prime}$ is the number of pairs of non-adjacent vertices receiving the same color, then

$$
2 \sqrt{\left(m+m_{c}^{\prime}\right)} \leq E_{c}(G) \leq 2\left(m+m_{c}^{\prime}\right)
$$

Proof. Consider,

$$
\begin{aligned}
\left(E_{c}(G)\right)^{2} & =\left(\sum_{i=1}^{n}\left|\lambda_{i}\right|\right)^{2} \\
& =\sum_{i=1}^{n}\left|\lambda_{i}\right|^{2}+\sum_{i \neq j}\left|\lambda_{i}\right|\left|\lambda_{j}\right| \\
& =\sum_{i=1}^{n}\left|\lambda_{i}\right|^{2}+2 \sum_{i<j}\left|\lambda_{i}\right|\left|\lambda_{j}\right| .
\end{aligned}
$$

By Lemma $5, S_{2}$ is the sum of all $2 \times 2$ principal minors of $A_{c}(G)$. Therefore, we get

$$
\begin{aligned}
\sum_{1 \leq i<j \leq n} \lambda_{i} \lambda_{j} & =\sum_{1 \leq i<j \leq n}\left|\begin{array}{ll}
a_{i i} & a_{i j} \\
a_{j i} & a_{j j}
\end{array}\right| \\
& =\sum_{1 \leq i<j \leq n}\left(a_{i i} a_{j j}-a_{i j} a_{j i}\right) .
\end{aligned}
$$

As $A_{c}(G)$ is the color matrix, $a_{i j}=a_{j i}$ and $a_{i i}=0 \forall i$. Thus,

$$
\begin{aligned}
\sum_{1 \leq i<j \leq n} \lambda_{i} \lambda_{j} & =\sum_{1 \leq i<j \leq n}-\left(a_{i j}\right)^{2} \\
& =-\left(m+m_{c}^{\prime}\right) .
\end{aligned}
$$

We know that

$$
\sum_{i<j}\left|\lambda_{i}\right|\left|\lambda_{j}\right| \geq\left|\sum_{i<j} \lambda_{i} \lambda_{j}\right|
$$


therefore, from Equation (9) and (10), we have

$$
\sum_{i<j}\left|\lambda_{i}\right|\left|\lambda_{j}\right| \geq\left|m+m_{c}^{\prime}\right| \cdot
$$

Using this together with Equations (2), (8) and (11), we get

$$
\begin{aligned}
\left(E_{c}(G)\right)^{2} & \geq 2\left|\left(m+m_{c}^{\prime}\right)\right|+2\left|\left(m+m_{c}^{\prime}\right)\right| \\
& \geq 4\left|\left(m+m_{c}^{\prime}\right)\right| .
\end{aligned}
$$

Taking positive square-root, we get

$$
E_{c}(G) \geq 2 \sqrt{\left(m+m_{c}^{\prime}\right)} .
$$

Now, for all connected graphs, $n \leq 2 m \leq 2\left(m+m_{c}^{\prime}\right)$.

Thus,

$$
\sqrt{2 n\left(m+m_{c}^{\prime}\right)} \leq \sqrt{4\left(m+m_{c}^{\prime}\right)^{2}} .
$$

Taking positive square-root, we get

$$
\sqrt{2 n\left(m+m_{c}^{\prime}\right)} \leq 2\left(m+m_{c}^{\prime}\right) .
$$

Therefore, from Equation (5), we can write

$$
E_{c}(G) \leq 2\left(m+m_{c}^{\prime}\right)
$$

and the result follows from Equations (12) and (13).

Remark 7 The inequality $2 \sqrt{\left(\mathrm{m}+\mathrm{m}_{\mathrm{c}}^{\prime}\right)} \leq \mathrm{E}_{\mathrm{c}}(\mathrm{G})$ is true for disconnected graphs also.

\section{$3 \quad$ Relationship between color energy and energy of a graph}

Although several aspects of color energy have been studied, relationship between color energy and energy was not taken into account. The color energy of $K_{n}$ is $2(n-1)$ which is same as its energy, whereas the color energy and the energy of $K_{1, n-1}$ are not same. So, it is interesting to find the relationship between color energy and energy and in this section an attempt is made to obtain this relationship. 
Theorem 8 If $\mathrm{G}$ is a graph of order $n$ and size $m$, then

$$
\left[\mathrm{E}_{\mathrm{c}}(\mathrm{G})\right]^{2} \geq \mathrm{E}(\mathrm{G}) \text {. }
$$

Proof. From Equations (1) and (5), we know that

$$
2 m \geq E(G) \text { and } E_{c}(G) \geq \sqrt{\left.2\left(m+m_{c}^{\prime}\right)+n(n-1) D^{\frac{2}{n}}\right)} .
$$

Therefore,

$$
\begin{aligned}
{\left[E_{c}(G)\right]^{2} } & \geq 2\left(m+m_{c}^{\prime}\right)+n(n-1) D^{\frac{2}{n}} \\
& \geq 2\left(m+m_{c}^{\prime}\right) \\
& \geq 2 m \\
& \geq E(G) .
\end{aligned}
$$

Next theorem tells us about the relation between $E_{c}(G), E(G)$ and the largest absolute value of the eigenvalue of the color matrix of $G$.

Theorem 9 If $\mathrm{G}$ is a colored graph and $\lambda_{\max }$ is the largest absolute value of the eigenvalue of the color matrix of $\mathrm{G}$, then $\mathrm{E}_{\mathrm{c}}(\mathrm{G}) \geq \frac{\mathrm{E}(\mathrm{G})}{\lambda_{\max }}$.

Proof. From Theorem 4, we have

$$
E_{c}(G) \geq\left(\frac{2\left(m+m_{c}^{\prime}\right)}{\lambda_{\max }}\right)
$$

Thus,

$$
\begin{aligned}
\lambda_{\max } E_{c}(G) & \geq 2\left(m+m_{c}^{\prime}\right) \\
& \geq 2 m \\
& \geq E(G), \text { by Equation (1). }
\end{aligned}
$$

Therefore,

$$
E_{c}(G) \geq \frac{E(G)}{\lambda_{\max }} .
$$

Computation of $E_{c}(G)$ and $E(G)$ have shown that $E(G) \leq E_{c}(G)$ and hence we state the following conjecture.

Conjecture 10 If $\mathrm{G}$ is a graph, then

$$
E(G) \leq E_{c}(G) .
$$




\section{Non-co-spectral color-equienergetic graphs}

The color-co-spectral graphs are the graphs having same color eigenvalues [1]. Obviously these graphs are color-equienergetic. It is interesting to note that $K_{n}$ and $K_{1, n-1}$ graphs are color-equienergetic with $E_{x}=2(n-1)$. However, they are not color-co-spectral.

In this section, we introduce a new family of unicyclic graphs which is colorequienergetic with some families of graphs and a family of bicyclic graphs which is color-hyperenergetic. Also we present explicit formulas for color energies of these graphs.

Theorem 11 If $\mathscr{S}=\mathrm{K}_{1, n-1}+\mathrm{e}$ is a unicyclic graph of order $\mathrm{n}$ and size $\mathrm{m}$ obtained by adding a single edge between two pendant vertices of the star graph $\mathrm{K}_{1, \mathrm{n}-1}$, then $\mathrm{E}_{\chi}(\mathscr{S})=2(\mathrm{n}-1)$.

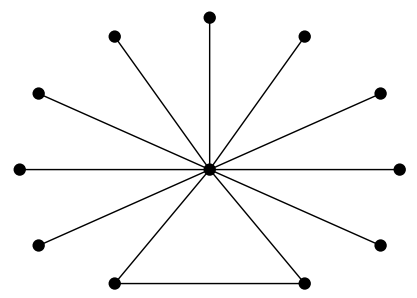

Figure $2: \mathscr{S}=\mathrm{K}_{1, n-1}+\mathrm{e}$

Proof. $\mathscr{S}$ is a unicyclic graph of order $n$ with $n-3$ pendant vertices. $\chi(\mathscr{S})=$ 3 , as it contains a $\mathrm{C}_{3}$.

The color matrix of $\mathscr{S}$ of order $n \times n$,

$$
A_{\chi}(\mathscr{S})=\left(\begin{array}{ccccccccc}
0 & 1 & 1 & -1 & -1 & -1 & \ldots & -1 & -1 \\
1 & 0 & 1 & 0 & 0 & 0 & \ldots & 0 & 0 \\
1 & 1 & 0 & 1 & 1 & 1 & \ldots & 1 & 1 \\
-1 & 0 & 1 & 0 & -1 & -1 & \ldots & -1 & -1 \\
-1 & 0 & 1 & -1 & 0 & -1 & \ldots & -1 & -1 \\
-1 & 0 & 1 & -1 & -1 & 0 & \ldots & -1 & -1 \\
\vdots & \vdots & \vdots & \vdots & \vdots & \vdots & \ddots & \vdots & \vdots \\
-1 & 0 & 1 & -1 & -1 & -1 & \ldots & 0 & -1 \\
-1 & 0 & 1 & -1 & -1 & -1 & \ldots & -1 & 0
\end{array}\right)_{n \times n}
$$

The characteristic polynomial

$$
\mathrm{P}_{\chi}(\mathscr{S}, \lambda)=\operatorname{det}\left(\lambda \mathrm{I}-\mathrm{A}_{\chi}(\mathscr{S})\right) .
$$


That is,

$$
P_{\chi}(\mathscr{S}, \lambda)=\left|\begin{array}{ccccccccc}
\lambda & -1 & -1 & 1 & 1 & 1 & \ldots & 1 & 1 \\
-1 & \lambda & -1 & 0 & 0 & 0 & \ldots & 0 & 0 \\
-1 & -1 & \lambda & -1 & -1 & -1 & \ldots & -1 & -1 \\
1 & 0 & -1 & \lambda & 1 & 1 & \ldots & 1 & 1 \\
1 & 0 & -1 & 1 & \lambda & 1 & \ldots & 1 & 1 \\
1 & 0 & -1 & 1 & 1 & \lambda & \ldots & 1 & 1 \\
\vdots & \vdots & \vdots & \vdots & \vdots & \vdots & \ddots & \vdots & \vdots \\
1 & 0 & -1 & 1 & 1 & 1 & \ldots & \lambda & 1 \\
1 & 0 & -1 & 1 & 1 & 1 & \ldots & 1 & \lambda
\end{array}\right|_{n \times n}
$$

In order to get the characteristic polynomial we apply a series of row transformations.

Adding first three rows of $\mathrm{P}_{\chi}(\mathscr{S}, \lambda)$ to take the factor $(\lambda-2)$ out of the first row.

$$
P_{\chi}(\mathscr{S}, \lambda)=(\lambda-2)\left|\begin{array}{ccccccccc}
1 & 1 & 1 & 0 & 0 & 0 & \ldots & 0 & 0 \\
-1 & \lambda & -1 & 0 & 0 & 0 & \ldots & 0 & 0 \\
-1 & -1 & \lambda & -1 & -1 & -1 & \ldots & -1 & -1 \\
1 & 0 & -1 & \lambda & 1 & 1 & \ldots & 1 & 1 \\
1 & 0 & -1 & 1 & \lambda & 1 & \ldots & 1 & 1 \\
1 & 0 & -1 & 1 & 1 & \lambda & \ldots & 1 & 1 \\
\vdots & \vdots & \vdots & \vdots & \vdots & \vdots & \ddots & \vdots & \vdots \\
1 & 0 & -1 & 1 & 1 & 1 & \ldots & \lambda & 1 \\
1 & 0 & -1 & 1 & 1 & 1 & \ldots & 1 & \lambda
\end{array}\right|_{n \times n}
$$

Adding first row of $\mathrm{P}_{\chi}(\mathscr{S}, \lambda)$ to its second row and taking the factor $(\lambda+1)$ out of the second row, we get

$$
P_{\chi}(\mathscr{S}, \lambda)=(\lambda-2)(\lambda+1)\left|\begin{array}{ccccccccc}
1 & 1 & 1 & 0 & 0 & 0 & \ldots & 0 & 0 \\
0 & 1 & 0 & 0 & 0 & 0 & \ldots & 0 & 0 \\
-1 & -1 & \lambda & -1 & -1 & -1 & \ldots & -1 & -1 \\
1 & 0 & -1 & \lambda & 1 & 1 & \ldots & 1 & 1 \\
1 & 0 & -1 & 1 & \lambda & 1 & \ldots & 1 & 1 \\
1 & 0 & -1 & 1 & 1 & \lambda & \ldots & 1 & 1 \\
\vdots & \vdots & \vdots & \vdots & \vdots & \vdots & \ddots & \vdots & \vdots \\
1 & 0 & -1 & 1 & 1 & 1 & \ldots & \lambda & 1 \\
1 & 0 & -1 & 1 & 1 & 1 & \ldots & 1 & \lambda
\end{array}\right|_{n \times n}
$$


Now adding second row of $\mathrm{P}_{\chi}(\mathscr{S}, \lambda)$ to its third row, we get

$$
P_{\chi}(\mathscr{S}, \lambda)=(\lambda-2)(\lambda+1)\left|\begin{array}{ccccccccc}
1 & 1 & 1 & 0 & 0 & 0 & \ldots & 0 & 0 \\
0 & 1 & 0 & 0 & 0 & 0 & \ldots & 0 & 0 \\
-1 & 0 & \lambda & -1 & -1 & -1 & \ldots & -1 & -1 \\
1 & 0 & -1 & \lambda & 1 & 1 & \ldots & 1 & 1 \\
1 & 0 & -1 & 1 & \lambda & 1 & \ldots & 1 & 1 \\
1 & 0 & -1 & 1 & 1 & \lambda & \ldots & 1 & 1 \\
\vdots & \vdots & \vdots & \vdots & \vdots & \vdots & \ddots & \vdots & \vdots \\
1 & 0 & -1 & 1 & 1 & 1 & \ldots & \lambda & 1 \\
1 & 0 & -1 & 1 & 1 & 1 & \ldots & 1 & \lambda
\end{array}\right|_{n \times n}
$$

Adding the third row of $\mathrm{P}_{\chi}(\mathscr{S}, \lambda)$ to its $\mathrm{q}^{\text {th }}$ row where $\mathrm{q} \geq 4$ and taking the factor $(\lambda-1)$ common from $q$ rows, we get

$$
P_{\chi}(\mathscr{S}, \lambda)=(\lambda-2)(\lambda+1)(\lambda-1)^{(n-3)}\left|\begin{array}{ccccccccc}
1 & 1 & 1 & 0 & 0 & 0 & \ldots & 0 & 0 \\
0 & 1 & 0 & 0 & 0 & 0 & \ldots & 0 & 0 \\
-1 & 0 & \lambda & -1 & -1 & -1 & \ldots & -1 & -1 \\
0 & 0 & 1 & 1 & 0 & 0 & \ldots & 0 & 0 \\
0 & 0 & 1 & 0 & 1 & 0 & \ldots & 0 & 0 \\
0 & 0 & 1 & 0 & 0 & 1 & \ldots & 0 & 0 \\
\vdots & \vdots & \vdots & \vdots & \vdots & \vdots & \ddots & \vdots & \vdots \\
0 & 0 & 1 & 0 & 0 & 0 & \ldots & 1 & 0 \\
0 & 0 & 1 & 0 & 0 & 0 & \ldots & 0 & 1
\end{array}\right|_{n \times n}
$$

Adding the first row and q rows of $\mathrm{P}_{\chi}(\mathscr{S}, \lambda)$ to its third row and subtracting the second row from its third row, then take the factor $[\lambda+(n-2)]$ out of the third row

$$
P_{\chi}(\mathscr{S}, \lambda)=(\lambda-2)(\lambda+1)(\lambda-1)^{(n-3)}[\lambda+(n-2)]\left|\begin{array}{ccccccccc}
1 & 1 & 1 & 0 & 0 & 0 & \ldots & 0 & 0 \\
0 & 1 & 0 & 0 & 0 & 0 & \ldots & 0 & 0 \\
0 & 0 & 1 & 0 & 0 & 0 & \ldots & 0 & 0 \\
0 & 0 & 1 & 1 & 0 & 0 & \ldots & 0 & 0 \\
0 & 0 & 1 & 0 & 1 & 0 & \ldots & 0 & 0 \\
0 & 0 & 1 & 0 & 0 & 1 & \ldots & 0 & 0 \\
\vdots & \vdots & \vdots & \vdots & \vdots & \vdots & \ddots & \vdots & \vdots \\
0 & 0 & 1 & 0 & 0 & 0 & \ldots & 1 & 0 \\
0 & 0 & 1 & 0 & 0 & 0 & \ldots & 0 & 1
\end{array}\right|_{n \times n}
$$


Now subtracting the second and third row of $\mathrm{P}_{\chi}(\mathscr{S}, \lambda)$ from its first row and subtract the third row from $q^{\text {th }}$ row where $q \geq 4$, we get

$$
\mathrm{P}_{\chi}(\mathscr{S}, \lambda)=(\lambda-2)(\lambda+1)(\lambda-1)^{(\mathrm{n}-3)}[\lambda+(\mathrm{n}-2)] \operatorname{det}(\mathrm{I})
$$

where I is the identity matrix.

Thus,

$$
P_{\chi}(\mathscr{S}, \lambda)=(\lambda-2)(\lambda+1)(\lambda-1)^{(n-3)}[\lambda+(n-2)] .
$$

Therefore,

$$
\operatorname{Spec}_{\chi} \mathscr{S}=\left(\begin{array}{cccc}
-(\mathrm{n}-2) & -1 & 1 & 2 \\
1 & 1 & \mathrm{n}-3 & 1
\end{array}\right)
$$

Hence, $\mathrm{E}_{\chi}(\mathscr{S})=2(\mathrm{n}-1)$.

Remark 12 We observe that, the family of graphs $\mathscr{S}, \mathrm{F}_{3}^{(\mathrm{k})}, \mathrm{K}_{\mathrm{n}}$ and $\mathrm{K}_{1, n-1}$ are color-equienergetic. From Equations (4), (6) and (14), clearly we can see their spectra are not same. So, these families of graphs are non-co-spectral colorequienergetic.

It is interesting to note that addition of an edge between a pendant vertex and a vertex of degree two to $\mathscr{S}$ brings a significant difference in its energy.

Theorem 13 If $\mathscr{H}=\mathscr{S}+\mathrm{e}$ is a bicyclic graph of order $\mathrm{n}$ and size $\mathrm{m}$ obtained by adding an edge between a pendant vertex and a vertex of degree two of the graph $\mathscr{S}=\mathrm{K}_{1, n-1}+e$, then $\mathrm{E}_{\chi}(\mathscr{H})=2(\mathrm{n}-3+\sqrt{5})$.

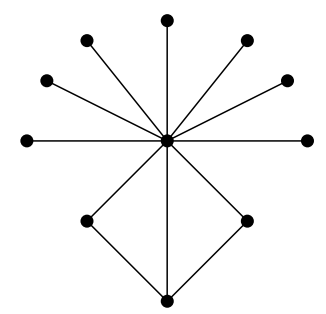

Figure $3: \mathscr{H}=\mathscr{S}+\mathrm{e}$

Proof. $\mathscr{H}$ is bicyclic graph of order $n$ with $n-4$ pendent vertices. Thus, $\chi(\mathscr{H})=3$, as it contains two copies of $\mathrm{C}_{3}$ with two vertices in common. 
The color matrix of $\mathscr{H}$ of order $n \times n$,

$$
A_{x}(\mathscr{H})=\left(\begin{array}{ccccccccc}
0 & 1 & 1 & -1 & -1 & -1 & \ldots & -1 & 1 \\
1 & 0 & 1 & 0 & 0 & 0 & \ldots & 0 & -1 \\
1 & 1 & 0 & 1 & 1 & 1 & \ldots & 1 & 1 \\
-1 & 0 & 1 & 0 & -1 & -1 & \ldots & -1 & 0 \\
-1 & 0 & 1 & -1 & 0 & -1 & \ldots & -1 & 0 \\
-1 & 0 & 1 & -1 & -1 & 0 & \ldots & -1 & 0 \\
\vdots & \vdots & \vdots & \vdots & \vdots & \vdots & \ddots & \vdots & \vdots \\
-1 & 0 & 1 & -1 & -1 & -1 & \ldots & 0 & 0 \\
1 & -1 & 1 & 0 & 0 & 0 & \ldots & 0 & 0
\end{array}\right)_{n \times n}
$$

The characteristic polynomial

$$
\mathrm{P}_{\chi}(\mathscr{H}, \lambda)=\operatorname{det}\left(\lambda \mathrm{I}-\mathrm{A}_{\chi}(\mathscr{H})\right) .
$$

That is,

$$
P_{\chi}(\mathscr{H}, \lambda)=\left|\begin{array}{ccccccccc}
\lambda & -1 & -1 & 1 & 1 & 1 & \ldots & 1 & -1 \\
-1 & \lambda & -1 & 0 & 0 & 0 & \ldots & 0 & 1 \\
-1 & -1 & \lambda & -1 & -1 & -1 & \ldots & -1 & -1 \\
1 & 0 & -1 & \lambda & 1 & 1 & \ldots & 1 & 0 \\
1 & 0 & -1 & 1 & \lambda & 1 & \ldots & 1 & 0 \\
1 & 0 & -1 & 1 & 1 & \lambda & \ldots & 1 & 0 \\
\vdots & \vdots & \vdots & \vdots & \vdots & \vdots & \ddots & \vdots & \vdots \\
1 & 0 & -1 & 1 & 1 & 1 & \ldots & \lambda & 0 \\
-1 & 1 & -1 & 0 & 0 & 0 & \ldots & 0 & \lambda
\end{array}\right|_{n \times n}
$$

Thus,

$$
\mathrm{P}_{\chi}(\mathscr{H}, \lambda)=[\lambda+(\mathrm{n}-3)](\lambda+\sqrt{5})(\lambda-\sqrt{5})(\lambda-1)^{(\mathrm{n}+1)}
$$

Therefore,

$$
\operatorname{Spec}_{\chi} \mathscr{H}=\left(\begin{array}{cccc}
-(\mathrm{n}-3) & -\sqrt{5} & 1 & \sqrt{5} \\
1 & 1 & (\mathrm{n}-3) & 1
\end{array}\right) .
$$

Hence, $E_{\chi}(\mathscr{H})=2(n-3+\sqrt{5})$.

Remark 14 The above theorem gives us the color energy of the family of graphs $\mathscr{H}=\mathscr{S}+\mathrm{e}$ of order $\mathrm{n}$ which is $2(\mathrm{n}-3+\sqrt{5})$. We observe that $\mathrm{E}_{\chi}(\mathscr{H})>2(\mathrm{n}-1)$. As a color-hyperenergetic graph has the color energy greater than $2(\mathrm{n}-1)$ [1], $\mathscr{H}$ is a family of color-hyperenergetic graphs. 


\section{Concluding remarks and scope}

In this study, we have explored new bounds for the color energy of graphs and have been successful in finding better bounds than those found in the literature. However, it remains as an open problem to determine graphs for which these bounds are sharp. Further, new bounds for $E_{c}(G)$ in terms of its order $\mathrm{n}$ can be determined. Another interesting area would be explore the relation between $E_{c}(G)$ and topological indices, and identify graphs for which they match. As we have observed the color energy is defined in terms of its color matrix which of course depends upon the coloring scheme. Therefore, study of color energy with respect to a specific coloring is yet another area to be investigated.

\section{References}

[1] C. Adiga, E. Sampathkumar, M. A. Sriraj, Shrikanth A. S., Color energy of a graph, Proc. Jangjeon Math. Soc., 16, 3 (2013) 335-351. $\Rightarrow$ 119, 120, 127, 131

[2] K. S. Betageri, The reduced color energy of graphs, J. Comp. and Math. Sci. 7, 1 (2016) $13-20 . \Rightarrow 121$

[3] K. S. Betageri and G. H. Mokashi, A note on reduced color energy of graphs, $J$. Comp. and Math. Sci. 7, 4 (2016) 203-212. $\Rightarrow 121$

[4] P. G. Bhat and S. D'souza, Color Laplacian energy of a graph, Proc. Jangjeon Math. Soc. 18, 3 (2015) 321-330. $\Rightarrow 121$

[5] P. G. Bhat and S. D'souza, Color signless laplacian energy of graphs, AKCE Int. J. of Graphs and Comb. (2017) http://dx.doi.org/10.1016/j.akcej.2017.02.003. $\Rightarrow 121$

[6] N. Biggs, Algebraic Graph Theory, Cambridge Univ. Press, Cambridge, UK, 1993. $\Rightarrow 121$

[7] S. A. Choudum, Graph theory, A NPTEL Course, (2012) http://nptel.ac.in/courses/111106050. $\Rightarrow 121$

[8] D. M. Cvetković, M. Doob, H Sachs, Spectra of Graphs- Theory and Application, Academic Press, New York, 1980. $\Rightarrow 121$

[9] I. Gutman, The energy of a graph, Ber. Math. Stat. Sekt. Forschungsz. Graz, 103 (1978) 1-22. $\Rightarrow 119,120$

[10] I. Gutman, The energy of a graph: old and new results, Combinatorics and Applications, A. Betten, A. Khoner, R. Laue and A. Wassermann, eds., Springer, Berlin, (2001) 196-211. $\Rightarrow 120$

[11] X. Li, Y. Shi and I. Gutman, Graph Energy, Springer, New York, 2012. $\Rightarrow 120$

[12] M. R. Rajesh Kanna, R. Pradeep Kumar and M. R. Farhani, Specific energies of friendship graph, Asian Acad Res J Multidiscip. 3, 1 (2016) 189-196. $\Rightarrow 121$ 
[13] M. R. Rajesh Kanna, R. Pradeep Kumar and R. Jagadeesh, Minimum covering color energy of a graph, Asian Acad Res J Multidiscip. 9, 8 (2015) 351-364. $\Rightarrow$ 121

[14] R. Rehman and I. C. F. Ipsen, Computing characteristic polynomials from eigenvalues, SIAM J. Matrix Anal. Appl. 32, 1 (2011) 90-114. $\Rightarrow 123$

[15] V. S. Shigehalli and K. S. Betageri, Color laplacian energy of graphs, J. Comp. and Math. Sci. 6, 9 (2015) 485-494. $\Rightarrow 121$

[16] V. S. Shigehalli and K. S. Betageri, A note on color energy graphs, Bull. Math. and Stat. Res. 4, 4 (2016) 47-50. $\Rightarrow 121$

[17] V. S. Shigehalli and K. S. Betageri, A note on color energy and color laplacian energy of graphs, Int. J. Math. Arch. 7, 10 (2016) 199-204. $\Rightarrow 121$

[18] D. B. West, Introduction to Graph Theory, Pearson, New Jersey, 2001. $\Rightarrow 121$

Received: August 28, 2017 • Revised: November 21, 2017 